LAWRENCE LIVERMORE N A TIO N A L LABORATORY

\section{Statistical Hot Spot Model for Explosive Detonation}

A. L. Nichols III

May 12, 2004

International Conference on New Models and Hydrocodes for Shock Wave Processes

College Park, MD, United States May 16, 2004 through May 20, 2004 
This document was prepared as an account of work sponsored by an agency of the United States Government. Neither the United States Government nor the University of California nor any of their employees, makes any warranty, express or implied, or assumes any legal liability or responsibility for the accuracy, completeness, or usefulness of any information, apparatus, product, or process disclosed, or represents that its use would not infringe privately owned rights. Reference herein to any specific commercial product, process, or service by trade name, trademark, manufacturer, or otherwise, does not necessarily constitute or imply its endorsement, recommendation, or favoring by the United States Government or the University of California. The views and opinions of authors expressed herein do not necessarily state or reflect those of the United States Government or the University of California, and shall not be used for advertising or product endorsement purposes. 
UCRL-

\title{
Statistical Hot Spot Model for Explosive Detonation
}

\author{
Albert L. Nichols III ${ }^{*}$ \\ Lawrence Livermore National Laboratory \\ Livermore, CA 94551
}

\begin{abstract}
The Non-local Thermodynamic Equilibrium Statistical Hot Spot Model (NLTE SHS), a new model for explosive detonation, is described. In this model, the formation, ignition, propagation, and extinction of hot spots is explicitly modeled. The equation of state of the explosive mixture is treated with a nonlocal equilibrium thermodynamic assumption. A methodology for developing the parameters for the model is discussed, and applied to the detonation velocity diameter effect. Examination of these results indicates where future improvements to the model can be made.
\end{abstract}

\section{Introduction}

A high explosive is an energetic material that is capable of releasing its stored energy in a time scale capable of sustaining a shock wave known as a detonation wave. The presence and need for energy localization in the ignition

\footnotetext{
* This work was performed under the auspices of the U.S. Department of Energy by University of California, Lawrence Livermore National Laboratory under Contract W-7405-Eng-48.
} 
and detonation of high explosives is a corner stone in our understanding of explosive behavior. This energy localization, known as hot spots, provides the match that starts the energetic response that is integral to the detonation.

Let us consider the processes that occur during the shock to detonation process. First, it is clear that the processes involved in ignition must be present during detonation, since the newly shocked material has no way of distinguishing between a shock loading from a flyer plate or a continuing detonation. The shock process deposits a significant amount of energy in the reactant. There will be two types of deposited energy: uniform and localized. The uniform energy deposition arises from the viscous dissipation of the shock progressing over uniform material. This process will heat the energetic material and can potentially cause reaction, if the shock is strong enough. Even when not detonating, the bulk temperature can be high enough to bulk reactivity to cause the complete decomposition of the explosive. However, for many explosives of interest, the shock required to detonate a uniform crystal would be stronger than that produced by the detonation of the explosive itself. This implies that in order for the detonation to progress, there must be another mechanism to increase the rate of energy release. Localized energy deposition, known as a hot spot, arises from a variety of defects in the explosive material. All defects are pre-existing, and can in principle be counted. Therefore, by understanding the number and type of defects, one could modify the formulation process of the explosive to change its properties. Finally, after the solid reactants have been transformed 
into gaseous intermediates, there can be longer diffusion controlled reactions that are required to explain certain long time behaviors.

In our model, we use the life cycle of a hot spot to predict explosive response. This life cycle begins with a random distribution of inhomogeneities in the explosive that we describe as a potential hot spot. A shock wave can transform these into hot spots that can then grow by consuming the explosive around them. The fact that the shock wave can collapse a potential hot spot without causing ignition is required in order to model phenomena like dead pressing. The burn rate of the hot spot is taken directly from experimental data. In our approach we do not assume that every hot spot is burning in an identical environment, but rather we take a statistical approach to the burning process. We also do not make a uniform temperature assumption in order to close the mixture equation of state, but track the flow of energy from reactant to product.

In Section II we define NLTE SHS model. In Section III we describe the process for defining the parameters used in the model. In Section IV we show results of the model as applied to the detonation velocity diameter effect, and conclude in Section V. 


\section{Non Local Thermodynamic Equilibrium Statistical Hot Spot Model}

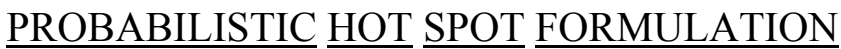

Nichols and Tarver ${ }^{1}$ initially described the statistical hot spot formulation. The first phase in constructing the statistical hot spot model is the consideration of the distribution of those hot spots. First, consider the probability $\mathrm{P}_{\mathrm{r}}$ that single hot spot of radius $\mathrm{R}$ will have reacted at a given location in a volume $\mathrm{V}$ in the explosive. This probability is given by:

$$
P_{r}=\frac{4 \pi R^{3}}{3 V}
$$

If there are $N_{R}$ of these hot spots randomly distributed in space, then the probability that a given location has not reacted $\mathrm{P}_{\mathrm{nr}}$ is simply the product of the independent probabilities. Assuming that the hot spots are independently located, $\mathrm{P}_{\mathrm{nr}}$ is defined as:

$$
P_{n r}=\left(1-\frac{4 \pi R^{3}}{3 V}\right)^{N_{R}}
$$

Taking the limit where the volume becomes large but the hot spot density remains fixed, Eq. (2) becomes:

$$
P_{n r}(R)=\exp \left(-\frac{4 \pi R^{3}}{3} \rho(R)\right)
$$


Finally, the combined probability of a region not having been reacted $\mathrm{P}_{\mathrm{nr}}(\mathrm{R})$ is simply the product of the probabilities associated with each hot spot radius. Therefore the final expression for $\mathrm{P}_{\mathrm{nr}}$ is:

$$
P_{n r}=\exp \left(-\frac{4 \pi}{3} \int_{0}^{\infty} R^{3} \rho(R) d R\right)
$$

The probability of not yet reacting is simply the mass fraction of the reactant in a reactive flow formulation. The probabilistic formulation makes it easier to consider a variety of different possibilities. For example, a similar line of reasoning can be used for two-dimensional hot spots (hotlines) and onedimensional hot spots (hotplanes). The latter would be useful for the modeling of shear banding as an ignition source, for example. If all of such ignition mechanisms could be defined, all that would be required for a complete hot spot model is to multiply their probability functions together.

\section{$\underline{\text { HOT SPOT DENSITY MODEL }}$}

The process derived in the previous section defines a mechanism for connecting the probability that some quantity of explosive has been reacted to the density distribution of hot spots. We define the probability density of the hot spots as:

$$
\begin{aligned}
& \rho(R, t)=\rho_{A}(R, t)+\rho_{D}(R, t) \\
& \rho_{A}(R, t)=\int_{-\infty}^{t} d \alpha \int_{t}^{\infty} d \omega \rho_{s}(\alpha, \omega) \delta\left(R-\varepsilon-\int_{\alpha}^{t} d \tau(\tau)\right)
\end{aligned}
$$




$$
\rho_{D}(R, t)=\int_{-\infty}^{t} d \alpha \int_{-\infty}^{t} d \omega \rho_{s}(\alpha, \omega) \delta\left(R-\varepsilon-\int_{\alpha}^{\omega} d \tau(\tau)\right)
$$

where $\rho s(\alpha, \omega)$ is the number density of hot spots that ignited at time $\alpha$, and died at time $\omega$. The Dirac-delta functions are used to define the size of the hot spot with the assumption that the initial hot spot size is $\varepsilon$, and that it then burns at a burn rate $\mathrm{v}$ out from that initial spot. The first term $\rho A(R, t)$ represents the population of hot spots of size $\mathrm{R}$ that are still growing (active) at time $\mathrm{t}$. The second term $\rho D(R, t)$ represents the population of hot spots of size $R$ that have stopped growing (died) by time $t$. It is important to remember that even though a hot spot may stop burning, the material that has burned within that hot spot must still be counted as reacted.

Let us now define the following projections of the density function:

$$
\begin{aligned}
& h(t)=\frac{4 \pi}{3} \int_{0}^{\infty} d R R^{3} \rho(R, t) \\
& \bar{g}(t)=\pi \int_{0}^{\infty} d R R^{2} \rho_{A}(R, t) \\
& \bar{f}(t)=2 \int_{0}^{\infty} d R R \rho_{A}(R, t) \\
& \bar{\rho}_{A}(t)=\int_{-\infty}^{t} d \alpha \int_{t}^{\infty} d \omega \rho_{s}(\alpha, \omega) \\
& \rho_{B}(t)=\int_{t}^{\infty} d \omega \rho_{s}(t, \omega)
\end{aligned}
$$

The $\mathrm{h}$ term is just the negative of the log of the probability defined in Eq. (4). The $\mathrm{g}$ and $\mathrm{f}$ terms are the two- and one-dimensional active projections of the density 
function, respectively. The number of hot spots that are active at time $t$ is $\bar{\rho}_{A}(t)$, and $\rho_{B}(t)$ is the number of hot spots created at time $t$. In the current model, it is assumed that all hot spots active at time thave the same rate of death $\mu(\mathrm{t})$, that is:

$$
\rho_{s}(\alpha, t)=\mu(t) \int_{t}^{\infty} \rho_{s}(\alpha, \omega)
$$

With these projections, it is possible to construct a set of differential equations to couple the high order reactant mass fraction with the much simpler active hot spot density.

$$
\begin{aligned}
\frac{\partial h}{\partial t} & =4 v(t) \bar{g}(t)+4 \pi \varepsilon^{3} \rho_{B}(t) / 3 \\
\frac{\partial \bar{g}}{\partial t} & =\pi v(t) \bar{f}(t)+4 \pi \varepsilon^{2} \rho_{B}(t)-\mu(t) \bar{g}(t) \\
\frac{\partial \bar{f}}{\partial t} & =2 v(t) \bar{\rho}_{A}(t)+2 \varepsilon \rho_{B}(t)-\mu(t) \bar{f}(t) \\
\frac{\partial \bar{\rho}_{A}}{\partial t} & =\rho_{B}(t)-\mu(t) \bar{\rho}_{A}(t)
\end{aligned}
$$

\section{PROPERTIES OF THE HOT SPOT DENSITY MODEL}

It is interesting to note some of the limits associated with the SHS model and compare them with the standard reactive flow models of Tarver. If we assume that the shock promptly ignites the hot spots, then the initial rate of fraction reacted $x$ will progress as $\dot{x} \propto x^{2 / 3}$. This is in accord with most of the reactive flow models. However, at long times, the rate of reaction will progresses as $\dot{x} \propto-(1-x) \ln (1-x)$. Note that this rate expression cannot be formulated as a power-law, as is the standard scheme in reactive flow rate laws. In fact, the SHS 
model is slower at finally consuming the reactant than any power law dependence less than 1 . On the other hand, a form factor reaction with coefficients $2 / 3$ and 0.7 , i.e. $\dot{x} \propto x^{2 / 3}(1-x)^{7}$, provides a good fit through the peak in the reactivity and is only slightly faster during the completion phase of the reaction.

\section{IGNITION MODEL}

In order to complete the set of equations defined in the previous section, we must define the rate at which hot spots are created. In order to model the explosive process, it is necessary to choose an ignition model that can encompass a variety of phenomena associated with high explosives. We begin by defining the initial density of potential hot spots $\rho_{P}{ }^{0}$. These potential hot spots can be anything from defects in the crystal lattice to voids in the region between the explosive grains. For the current model, we only limit ourselves in that the potential hot spot must transform into a roughly spherical hot spot. Most postulated hot spot formation mechanisms involving void collapse predict that the spherical hot spots form upon full collapse ${ }^{2}$. Other potential hot spot formation mechanisms, such as shear banding, would transform into roughly planar hot spots and thus are not considered in this treatment. The shock process compresses these potential hot spots. If they are compressed to a sufficiently high temperature, they will start to react (a hot spot). If the process is too weak, then the potential hot spot will be destroyed without creating a hot spot. Without 
such a process, any sufficient compression of the explosive would lead to reaction, even that from an isostatic press. However, since explosives do have strength, there must be sufficient force to overcome that internal void strength before any changes to the potential hot spot density can occur. The following phenomenological ignition model captures these features.

$$
\begin{aligned}
& \mathrm{K}(p)=\left(\frac{A P^{*}\left(p-P_{0}\right)}{P^{*}+p-P_{0}}\right) H\left(p-P_{0}\right) \\
& \dot{\rho}_{P}=-\rho_{P} \mathrm{~K}(p) \\
& \rho_{B}=\rho_{P}\left(\mathrm{~K}(p)-\mathrm{K}\left(P_{A}\right)\right) H\left(\mathrm{~K}(p)-\mathrm{K}\left(P_{A}\right)\right)
\end{aligned}
$$

Here $\mathrm{K}(\mathrm{p})$ is the rate of potential hot spot transformation, and $\mathrm{K}\left(\mathrm{P}_{\mathrm{A}}\right)$ is the constant death rate for potential hot spots. It is the rate of transformation at the pressure $P_{\mathrm{A}}$ when the first hot spots actually start igniting. $P_{0}$ is the ignition rate threshold pressure that represents the internal resistance to void collapse. To prevent unrealistically large collapse rates during numerical pressure spikes, $P^{*}$ is defined as the saturation pressure. $H$ is the heavy side step function, which is zero for all arguments less than zero and one for everything else. We originally envisioned a compression rate dependent ignition rate, but such a rate can be extremely mesh-size dependent. More complex ignition models can be formulated as this model evolves. 


\section{$\underline{\text { NON-LOCAL THERMODYNAMIC EQUILIBRIUM CHEMICAL MATERIAL }}$}

\section{MODEL}

Having defined the mechanism for reaction of the explosive, we need to define the model for the equation of state of the mixture of reactants and products. In our model, the extent of composition change and the hydrodynamic work are conducted simultaneously and self consistently. At the beginning of a time step, the state of the material is given by the mass fractions $\left\{x_{i}\right\}$, specific energies (energy per reference volume) $\left\{\mathrm{e}_{\mathrm{i}}\right\}$, and specific relative volumes (volume per reference volume) $\left\{\mathrm{v}_{\mathrm{i}}\right\}$ of each species $\mathrm{i}$. We also can define the total energy and volume of the system:

$$
\begin{aligned}
& e=\rho^{0} \sum_{i} e_{i} x_{i} / \rho_{i}^{0} \\
& v=\rho^{0} \sum_{i} v_{i} x_{i} / \rho_{i}^{0}
\end{aligned}
$$

where $\rho^{0}$ is the reference density of the chemical material, and $\rho_{i}^{0}$ is the reference density of the $i^{\text {th }}$ species. We need to follow the energy of the system through the reactivity, heat and work phases. By defining $\mathrm{x}_{\mathrm{ij}}$ as the change increase in the mass fraction of the $\mathrm{i}^{\text {th }}$ species that came from the $\mathrm{j}^{\text {th }}$ species, we have that the mass fraction change is

$$
\Delta x_{i}=\sum_{j} x_{i j}-\sum_{j} x_{j i}
$$

The NLTE chemical material model is solved implicitly. Several passes are conducted until self-consistency in the extent of reaction and pressure 
equilibrium between all of the species is achieved. Each pass is broken into four phases. The first phase calculates the extent of reaction based on the average of the initial and final pressures and temperatures of the previous pass. For the initial pass, only the initial condition is used to determine the rates. The second phase imputes half of the composition change. The splitting of the application of the composition change before and after the work phase is what allows this model to maintain second order accuracy. The mass, energy, and volume of each of the reacting species is moved from the reactant to the product. So for the mass, volume, and energy we get the following set of equations:

$$
\begin{aligned}
x_{i}^{I I} & =x_{i}^{t}-\frac{1}{2} \sum_{j} x_{j i}+\frac{1}{2} \sum_{j} x_{i j} \\
\frac{e_{i}^{I I} x_{i}^{I I}}{\rho_{i}^{0}} & =\frac{e_{i}^{t} x_{i}^{t}}{\rho_{i}^{0}}-\frac{1}{2} \sum_{j} x_{j i} \frac{e_{i}^{t}}{\rho_{i}^{0}}+\frac{1}{2} \sum_{j} x_{i j} \frac{e_{j}^{t}}{\rho_{j}^{0}} \\
\frac{v_{i}^{I I} x_{i}^{I I}}{\rho_{i}^{0}} & =\frac{v_{i}^{t} x_{i}^{t}}{\rho_{i}^{0}}-\frac{1}{2} \sum_{j} x_{j i} \frac{v_{i}^{t}}{\rho_{i}^{0}}+\frac{1}{2} \sum_{j} x_{i j} \frac{v_{j}^{t}}{\rho_{j}^{0}}
\end{aligned}
$$

It is important to note that the process from going from state " $\mathrm{t}$ " to state "II" conserves total mass, volume and energy. It can be thought of separating an appropriate amount of mass with its energy and volume and transferring it to that of the receiving species.

The third phase of each pass changes the volume of each species while holding the composition fixed. This last constraint implies $x_{i}{ }_{i}^{I I}=x_{i}{ }_{i}^{I I I}$. Thus, with $\omega_{i}$ the external heat source, we have for each species: 


$$
e_{i}^{I I I}=e_{i}^{I I}+\omega_{i}-\int_{v_{i}^{I I}}^{v_{i}^{I I I}}\left(p_{i}+q_{i}\right) d v_{i}
$$

where $p_{i}$ is the pressure and a function of the energy and volume, and $q_{i}$ is the artificial viscosity as determined by the size of the element, the change in the relative volume, the sound speed, and the time step length. In general, it has been found that the species $q$ factors should be turned off, as they can give rise to extremely high temperatures during various stages of the iteration. These high temperatures can lead to large reaction rates, causing even more relative volume change for the reacting species. These two effects compound each other to cause a numerical instability, especially if one includes a quadratic $q$ for the species. This discussion does not apply to the $\mathrm{q}$ applied to the element, but only to that specifically related to the species.

Finally, the fourth phase of each pass imputes the final half of the composition change, again with overall conservation of mass, volume, and energy:

$$
\begin{aligned}
x_{i}^{t+\Delta t} & =x_{i}^{I I I}-\frac{1}{2} \sum_{j} x_{j i}+\frac{1}{2} \sum_{j} x_{i j} \\
\frac{e_{i}^{t+\Delta t} x_{i}^{t+\Delta t}}{\rho_{i}^{0}} & =\frac{e_{i}^{I I I} x_{i}^{I I I}}{\rho_{i}^{0}}-\frac{1}{2} \sum_{j} x_{j i} \frac{e_{i}^{I I I}}{\rho_{i}^{0}}+\frac{1}{2} \sum_{j} x_{i j} \frac{e_{j}^{I I I}}{\rho_{j}^{0}} \\
\frac{v_{i}^{t+\Delta t} x_{i}^{t+\Delta t}}{\rho_{i}^{0}} & =\frac{v_{i}^{I I I} x_{i}^{I I I}}{\rho_{i}^{0}}-\frac{1}{2} \sum_{j} x_{j i} \frac{v_{i}^{I I I}}{\rho_{i}^{0}}+\frac{1}{2} \sum_{j} x_{i j} \frac{v_{j}^{I I I}}{\rho_{j}^{0}}
\end{aligned}
$$

We also require that the internal energy change calculated by the sum of the species processes be equal to the change of the total internal energy. That implies the following requirement: 


$$
\omega-\int_{v^{t}}^{v^{t+\Delta t}}(p+q) d v=\sum_{i} \frac{\rho^{0}}{\rho_{i}^{0}} x_{i}^{I I} \omega_{i}-\sum_{i} \frac{\rho^{0}}{\rho_{i}^{0}} x_{i}^{I I} \int_{v_{i}^{t+\Delta t}}^{v_{i}^{t+\Delta t}}\left(p_{i}+q_{i}\right) d v_{i}
$$

The artificial viscosity $q$ will be divided into two parts: $q^{e}$ the artificial viscosity derived from the element motion, and $\mathrm{q}^{\mathrm{i}}$ that derived from internal processes. In our current model, the assumption is made that the external energy and $\mathrm{q}^{\mathrm{e}} \mathrm{dv}$ work associated with the zone is distributed equally to each species by their mass:

$$
\omega_{i}=\frac{\rho_{i}^{0}}{\rho^{0}}\left(\omega-\int_{v^{t}}^{v^{t+\Delta t}} q^{e} d v\right)
$$

Once the energy and volume have been calculated for the " $t+\Delta t$ " state, we calculate the pressure and temperature using the equation of state for each species. These state variables are used to calculate the reaction rates in the next phase 1 . Note that these phases define the process by which the NLTE chemical element proceeds from its initial state to its final state. We have still not completely defined the path however. In particular, the relative volume at state "III" is still a free parameter within the constraint that the total volume sum must still be satisfied. However, it is these values that are directly tied to the final volume and energy that is used to calculate the pressure. We require pressure equilibrium in the NLTE chemical material at the end of the time step. Therefore, it is necessary to determine how the change of the state "III" volume changes the final species pressure. Using a the standard Newton-Raphson solution technique for pressure equilibration, we have that the equilibrium pressure $P^{t+\Delta t}$ is: 


$$
\begin{gathered}
p^{t+\Delta t}=\frac{\sum_{i j} \frac{x_{i}^{I I I}}{\rho_{i}^{0}}\left[\frac{\partial \underline{p}}{\partial \underline{\underline{v}}}\right]_{i j}^{-1} p_{j}^{t+\Delta t}}{\sum_{i j} \frac{x_{i}^{I I I}}{\rho_{i}^{0}}\left[\frac{\partial \underline{p}}{\partial \underline{v}}\right]^{-1} p_{i j}^{t+\Delta t}} \\
{ }^{\text {New }} \underline{v}^{I I I}={ }^{\text {Old }} \underline{v}^{I I I}+\left[\frac{\partial \underline{p}}{\partial \underline{v}^{I I I}}\right]\left(p^{t+\Delta t} \underline{1}^{-p^{t+\Delta t}}\right)
\end{gathered}
$$

where variables with an underline are vectors of the species values. The kernel of this formula is the matrix of the change in the pressure of the $i^{\text {th }}$ species with the change in the relative volume of the $j^{\text {th }}$ species. Given that the number of species in this model is arbitrary, that the indicated matrix inverse would be number of species by number of species, and that the non-diagonal contributions go to zero as the change in concentration goes to zero, we replace the matrix inversion with its zero reaction limit. In order to insure iterative convergence, the maximum extent of reaction during any time step must be limited. This is done by time step constraint. With this simplification, the $\partial \underline{p} / \partial \underline{v}$ term becomes diagonal and that for the $j^{\text {th }}$ species becomes:

$$
\frac{\partial p_{j}}{\partial v_{j}}=\left(\frac{\partial p_{j}}{\partial v_{j}}\right)_{e_{j}}-\left(\frac{\partial p_{j}}{\partial e_{j}}\right)_{v_{j}}\left[p_{j}^{I I I}+q_{j}\left(v_{j}^{I I I}-v_{j}^{I I}\right)+\int_{v_{j}^{I I}}^{v_{j}^{I I I}} \frac{\partial q_{j}}{\partial v_{j}^{I I I}}\right]
$$

Finally, the last variable that needs to be identified is the internal artificial viscosity $q^{i}$. For that we note that $p+q$ at the end of the time step for the entire element needs to be the appropriate sum of those for each component as work was begin performed. Thus: 


$$
q^{i}=\sum_{j}\left[x_{j}^{I I I} \frac{v_{j}^{I I I} \rho^{0}}{v^{t+\Delta t} \rho_{j}^{0}}\left(p_{j}^{I I I}+q_{j}^{I I I}\right)\right]-p^{t+\Delta t}
$$

Having described the process for each pass, the code iterates on the $\mathrm{v}_{\mathrm{j}}^{\mathrm{III}}$ until pressure and reaction equilibration have occurred.

\section{Model Parameterization}

We have defined a total of 8 parameters, not counting those associated with the equation of state, for the statistical hot spot model. They are: $\mathrm{P}_{0}, \mathrm{P}^{*}, \mathrm{~A}, \mu$, $v, \mathrm{P}_{\mathrm{A}}, \rho_{P}{ }^{0}$, and $\varepsilon . \mathrm{P}_{0}$ is clearly related to the yield strength of the explosive, and so we will use the yield strength in our model. The burn velocity $v$ can be experimentally determined by any of the standard burn rate measurement techniques, such as strand-burner and diamond anvil experiments. The value of $\mathrm{P}_{\mathrm{A}}$ should be chosen to match explosive shock recovery experiments, so that the value of $P_{A}$ is set equal to the value of the shock pressure that just begins to ignite the explosive.

A heuristic argument can be used to determine $\rho_{P}{ }^{0}$, and $\varepsilon$. If one assumes that the total initial hot spot volume will equal the initial void volume and that enough hot spots need to be created so that when they burn with velocity $v$ at the detonation pressure $p_{D}$, they will consume the entire explosive in the reaction zone time $\tau$ and initial void density $\rho_{v}$, then: 


$$
\begin{aligned}
\rho_{v} & \approx 4 \pi \varepsilon^{3} \rho_{P}^{0} / 3 \\
\rho_{A}^{0} & \approx \rho_{P}^{0}\left[1-K\left(P_{A}\right) / K\left(p_{D}\right)\right] \\
1 & \approx 4 \pi(\varepsilon+v \tau)^{3} \rho_{A}^{0} / 3
\end{aligned}
$$

Another heuristic argument can be applied to the ignition pre-factor A and $\mathrm{P}^{*}$. It is reasonable to assume that the rate of collapse of the void regions is proportional to the product of the void radius and the particle velocity $u$ in the shock wave. For relatively low pressures, we can Taylor expand the volume change using the adiabatic compressibility, and get:

$$
\begin{aligned}
R & \approx \frac{u}{2 \varepsilon} \approx \frac{1}{2 \varepsilon} \sqrt{\frac{\left(p-P_{0}\right)\left(\rho-\rho_{0}\right)}{\rho \rho_{0}}} \\
& \approx\left(p-P_{0}\right) /\left(2 \varepsilon \rho_{0} c\right)
\end{aligned}
$$

where $\rho_{0}$ is the initial density, and $c$ is the reference sound speed. Comparing to the ignition definition in the limit of small $\left(\mathrm{p}-\mathrm{P}_{0}\right)$, we find

$$
A \approx \frac{1}{\left(2 \varepsilon \rho_{0} c\right)}
$$

Although it might be tempting to use an ignition form defined by the particle velocity, the complexities associated with making it work under the variety initial conditions would be daunting. For example, changes in temperature would necessitate a change in the reference density within the root sign. Without such a change, hot systems could never ignite. We can handle the natural curvature that comes out of this formulation by an appropriate choice of $P^{*}$. 
Reactant and product equations of state are needed to describe the states attained during shock compression. The Jones-Wilkins-Lee (JWL) equation of state is used for the reactant with typical parameters for an HMX-based plastic bonded explosive.

$$
P=A\left(1-\frac{\omega}{R_{1} V}\right) e^{-R_{1} V}-B\left(1-\frac{\omega}{R_{12} V}\right) e^{-R_{2} V}+\frac{\omega E}{V}
$$

where $\mathrm{P}$ is pressure, $\mathrm{V}$ is relative volume, $\mathrm{E}$ is the internal energy, $\omega$ is the Gruneisen coefficient, and $A, B, R_{1}$, and $R_{2}$ are constants. For a typical HMXbased plastic bonded explosive, the initial density is $1.85 \mathrm{~g} / \mathrm{cm}^{3}, \mathrm{R}_{1}=14.1, \mathrm{R}_{2}=$ 1.41, $\omega=0.8938, A=9522 \mathrm{Mbar}$, and $\mathrm{B}=0.05944 \mathrm{Mbar}$. This JWL equation fits the measured reactant Hugoniot data at low shock pressures and the von Neumann spike data at high pressures ${ }^{3}$. The reaction products are described by LEOS tables fit to product equation of state calculated by the CHEETAH chemical equilibrium code ${ }^{4}$.

\section{Results}

As discussed in the previous section, the ignition and growth of reaction model has eight parameters: $\mathrm{P}_{0}, \mathrm{P}^{*}, \mathrm{~A}, \mu, v, \mathrm{P}_{\mathrm{A}}, \rho_{P^{0}}$, and $\varepsilon$. The parameters for the model developed here are listed in Table $1 . \mathrm{P}_{0}$ is the ignition rate threshold pressure and is set to the Hugoniot elastic limit for HMX ${ }^{5}$. The activation threshold has been set to twice the Hugoniot elastic limit. The reaction growth rate $v$ is assumed to be a function of pressure as measured experimentally in 
strand burner ${ }^{6}$ and diamond anvil cells on pure HMX7. This pressure versus burn rate function is shown in Table 1. The initial hot spot diameter $\varepsilon$ and initial number of potential hot spot sites $\rho_{P}^{0}$ are derived from the burn rate at the detonation conditions based on a reaction time set to match experimental results.

We examine the detonation velocity diameter effect with the statistical hot spot model. In order to determine the detonation velocity, two-dimensional axisymmetric problems at the requisite diameters were created. The length to diameter ratio was set to 4 , and the calculation was run for 10 microseconds times the radius in $\mathrm{cm}$. This generally ensured that the shock wave has proceeded through approximately $90 \%$ of the length. The cylinder of explosive was given a velocity of $.1 \mathrm{~mm} /$ microsecond into unmovable stonewall. A mesh resolution of 1000 elements per $\mathrm{cm}$ was used for most of the work shown here. Multiple resolutions were used to confirm mesh convergence. In order to capture the locus of the detonation front, the cylinder was divided into two regions, one a single element thick running along the axis. The location of the highest pressure in this region was then written to a history file for processing. The detonation velocity was calculated by a least squares fit to the final eighth of the time steps.

Two models are shown in Table 1. The first model developed uses a burn rate pressure exponent of $\sim 0.75$ that essentially matches the experimental data. The second model follows the higher-pressure burn rate and then continues with 
an exponent of 2 to the detonation pressure. The detonation velocity versus cylinder diameter for both models and the experimental results of A. W. Campbell and Ray Engelke ${ }^{8}$ are plotted in Figure 1. Although model 1 reproduces the detonation velocity diameter effect for large diameters, the detonation continues to propagate even at small diameters, contrary to experimental observation. This behavior is typical of all reaction models that use a pressure burn rate exponent of 0.75 . Essentially, the burn rate does not change rapidly enough to cause the classical detonation failure, but instead the detonation velocity steadily drops until it merges with the sound speed. The fact that this model does not properly fail at small diameters leads one to believe that there are processes that are not being captured in this model. Another interesting feature is that for the model 1 like systems, the function of the detonation velocity to inverse diameter is essentially a straight line.

On examination of the results for model 1, it became clear that some other process needed be present to reproduce the experimental results. One such mechanism would be the bulk heating of the explosive by the detonation wave. To test this idea, we augmented the hot spot reaction with thermal reactions based on the reaction rate parameters of Tarver et. al. ${ }^{9}$. For the current equation of state system, the non-linearity of the thermal reaction rated caused the reactants to transform into products at rates substantially higher than needed. Aside from causing a significant reduction in the time step, this mechanism led to significant numerical instabilities due to the non-linearity of the chemistry. 
One issue that could affect the model is the burn rate function. The experimental data that we use was collected at room temperature. Although the burn rate tends to exhibit weak temperature dependence, temperature changes in the order of a thousand degrees will probably result in significant change in the burn rate. An effective burn rate with a higher-pressure exponent would represent the temperature increased burn rate, since the temperature increases as we increase the shock pressure. This is the basis of model 2. Model 2 slightly under predicts the detonation velocity in the intermediate diameters until just before detonation failure. The model does reproduce the classic detonation failure diameter.

\section{Conclusion}

In this paper, we have described a new detonation model for HMX. The equations of state models are based on current best practice. The reaction parameters have been based available reactant experimental data. The mixture equation of state equilibrates the pressure of each species, but does not equilibrate the temperature. Instead, we track the flow of energy as the composition changes from one species to another. These keeps the reactants cold while the products will be hot, in keeping with the physical model. These models were applied to the detonation velocity diameter effect, to good result.

One issue that needs to be addressed is equation of state of the reactant species at shock conditions. The standard schemes for calibrating the EOS of 
reactants are capable of determining the pressure at shock compression, but the temperature is more difficult. An improved EOS for temperature is necessary to add a bulk reactivity contribution to the detonation model.

Further investigation into the effect of multiple hot spot sizes should be conducted. A common postulate is that small voids would be more difficult to ignite than large voids. Higher shock pressures would ignite significantly more hot spots than lower pressure. This would increase the effective burn rate at high pressure, as there would be more sites to burn the reactant. As was pointed out, a higher-pressure exponent is necessary to reproduce the detonation failure data.

Finally, although we have focused on the high pressure end of shock response, it should be pointed out that these models are capable of modeling the process known as dead pressing, where an initial low amplitude shock desensitizes the explosive, making it more difficult for a second shock wave to transition to detonation.

\section{Acknowledgments}

The author would like to thank Craig Tarver, Jack Reaugh, Brad Wallin and Melvin Baer for useful discussions. 
Table 1. Reaction Rate Parameters

\begin{tabular}{|c|c|c|}
\hline Parameter & Value (Model 1) & Value (Model 2) \\
\hline$P_{0}(\mathrm{GPa})$ & 0.6 & 0.6 \\
\hline$P_{A}(\mathrm{GPa})$ & 1.2 & 1.2 \\
\hline$P^{*}(\mathrm{GPa})$ & 10 & 10 \\
\hline Void Fraction & 0.02 & 0.02 \\
\hline$\tau(\mu s)$ & 0.009 & 0.009 \\
\hline$\varepsilon(\mathrm{cm})$ & $8.63581 E-06$ & $1.0531 \mathrm{e}-4$ \\
\hline$\rho p^{0}\left(\mathrm{~cm}^{-3}\right)$ & $7.41266 \mathrm{E}+12$ & $4.087101054 \mathrm{e} 9$ \\
\hline$A(\mathrm{~cm}-\mu \mathrm{s} / \mathrm{g})$ & 51741.2662 & 4242.78383 \\
\hline$D\left(\mu s^{-1}\right)$ & 292.8750917 & 24.0157575 \\
\hline$\mu\left(\mu \mathrm{s}^{-1}\right)$ & 1 & 1 \\
\hline $\begin{array}{l}\text { Pressure } \\
\text { (GPa) }\end{array}$ & $\begin{array}{l}\text { Burn Rate } \\
(\mathrm{cm} / \mu \mathrm{s})\end{array}$ & $\begin{array}{l}\text { Burn Rate } \\
(\mathrm{cm} / \mu \mathrm{s})\end{array}$ \\
\hline 0.0001 & $2.35 \mathrm{E}-07$ & $2.35 \mathrm{E}-07$ \\
\hline 0.1 & $5.00 \mathrm{E}-05$ & $5.00 \mathrm{E}-05$ \\
\hline 3. & $7.00 \mathrm{E}-04$ & $7.00 \mathrm{E}-04$ \\
\hline 7.5 & & $2.50 \mathrm{E}-03$ \\
\hline 10 & & 4.00E-03 \\
\hline 15. & & $9.00 \mathrm{E}-03$ \\
\hline 20 & $2.85 \mathrm{E}-3$ & \\
\hline 37. & $4.1 \mathrm{E}-3$ & $5.00 \mathrm{E}-02$ \\
\hline 100. & & $5.00 \mathrm{E}-02$ \\
\hline 200 & $1.6 \mathrm{E}-2$ & \\
\hline
\end{tabular}


Figure 1. Detonation Velocity as a function of inverse cylinder diameter. The solid square dots are the experimental results of Campbell and Engelke. Model 1 has a explosive burn rate exponent of approximately 0.75 , while model two has a higher exponent above $3 \mathrm{GPa}$.

\section{Detonation Velocity}

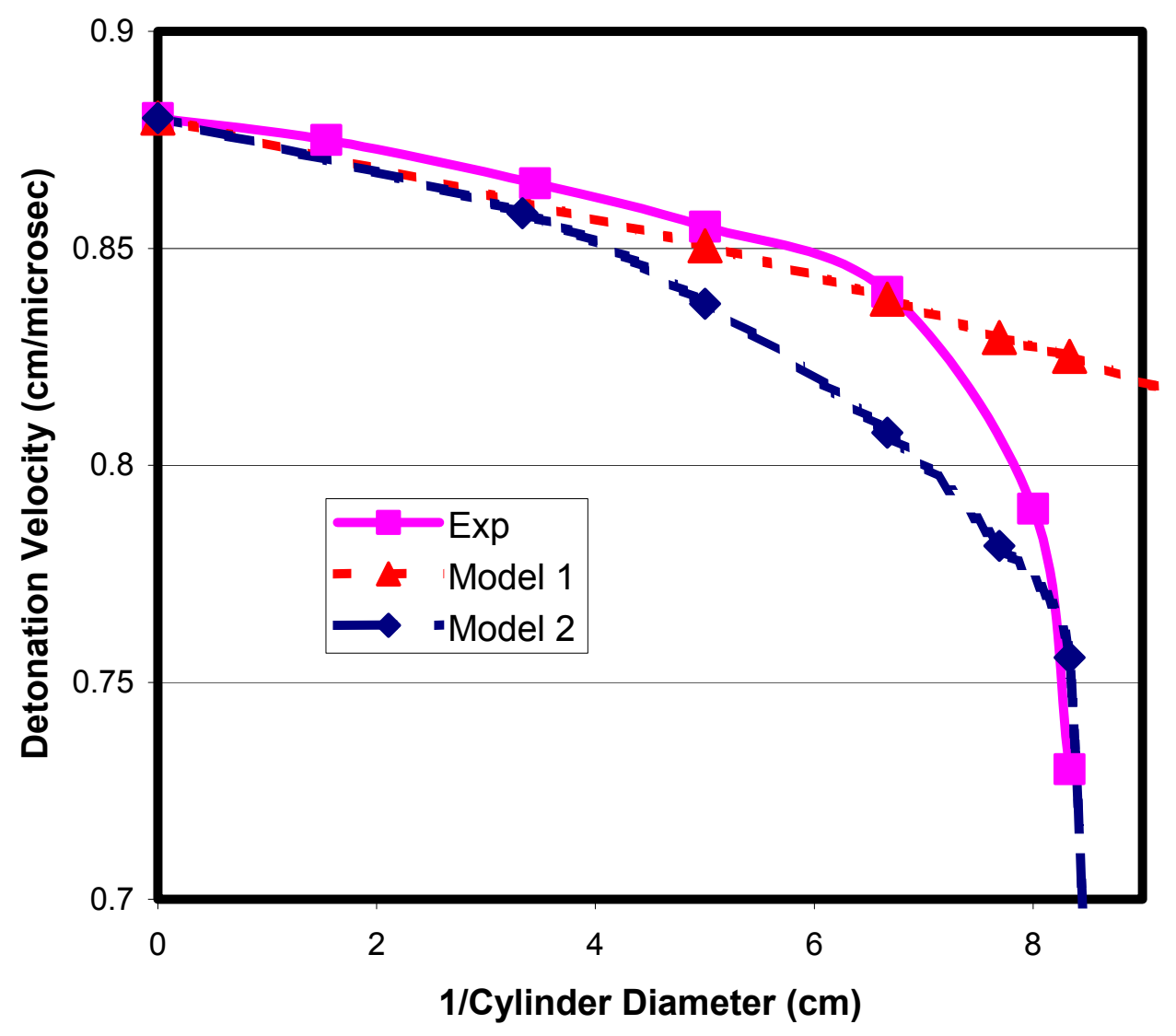




\section{References:}

1. Nichols, A. L., III, and Tarver, C. M., presented at Twelfth International Detonation, San Diego, CA, 2002.

2. Conley, P. A., Benson, D. J., and Howe, P. M., Eleventh International Detonation Symposium, Office of Naval Research ONR 33300-5, Snowmass, CO, 1998, pp. 768-780.

3. Tarver, C. M., Urtiew, P. A., Chidester, S. K., and Green, L. G., Propellants, Explosives, Pyrotechnics 18, 117 (1993).

4. Fried, L., Howard, W. M., and Souers, P. C., presented at Twelfth International Detonation, San Diego, CA, 2002.

5 . Dick, J. J., and Nartunez, A. R., Shock Compression of Condensed Matter2001, Furnish, M. D., Thadhani, N. N., and Horie, Y, eds. CP-620, AIP Press, New York, 2002, pp 817.

6. Maienschein, J. L. and Chandler, J. B., Eleventh International Detonation Symposium, Office of Naval Research ONR 33300-5, Snowmass, CO, 1998, pp. 872-879.

7. Farber, D. L., Zaug, J. M., and Ruddle, C., Shock Compression of Condensed Matter-2001, Furnish, M. D., Thadhani, N. N., and Horie, Y, eds. CP-620, AIP Press, New York, 2002. 
8. A. W. Campbell and Ray Engelke, 6th Symposium (International) on Detonation, White Oak, MD, August 1976

9. Tarver, C. M., Chidester, S. K., and Nichols, A. L. III, 1996, J. Phys. Chem. 100, 5794. 